\title{
A Novel Standard for Hepatocellular Carcinoma Screening Intensity After Hepatitis C Elimination
}

\author{
Akio Miyasaka $\mathbb{D}^{1,2}$ \\ Yuichi Yoshida ${ }^{1,2}$ \\ Akiko Suzuki ${ }^{1}$ \\ Kei Sawara ${ }^{1,3}$ \\ Yasuhiro Takikawa' \\ 'Division of Hepatology, Department of \\ Internal Medicine, Iwate Medical \\ University School of Medicine, Shiwa-gun, \\ Iwate, Japan; ${ }^{2}$ Department of \\ Gastroenterology, Iwate Prefectural \\ Ninohe Hospital, Ninohe, Iwate, Japan; \\ ${ }^{3}$ Department of Gastroenterology, Iwate \\ Prefectural Kamaishi Hospital, Kamaishi, \\ Iwate, Japan
}

Purpose: To investigate long-term incidence of hepatocellular carcinoma (HCC) and the factors associated with HCC occurrence after achieving sustained virological response (SVR) by direct-acting antiviral agent (DAA) treatment for hepatitis $\mathrm{C}$ virus (HCV).

Methods: A total of 476 patients (male 227, female 249; median age 68) with chronic HCV infection who were treated with DAAs and achieved SVR were analyzed. The incidence of HCC and factors related to the development of HCC after HCV elimination were evaluated. Results: The median observation period was 46.4 months. During this period, 40 patients developed HCC. The incidence rates of HCC were 3.7\%, 6.0\%, 7.1\%, 9.3\%, and $10.6 \%$ at 1, 2, 3, 4 , and 5 years post-SVR12, respectively. Multivariate analysis with pre-treatment factors revealed that platelet count, $\alpha$-fetoprotein, fibrosis-4 (Fib-4) index, and previous HCC history were independent factors that contributed to development of HCC post-SVR following DAA treatment. Of these factors, previous HCC history was the most significant, followed by Fib-4 index. Using these two factors, a novel scoring system was established. The presence of previous HCC history was scored as 2, and then, the absence of previous HCC history was stratified by Fib-4 index $(\geq 3.07,1 ;<3.07,0)$. The HCC occurrence rate at 5 years was $0.4 \%$ in the 0 -point group, $6.8 \%$ in the 1-point group, and $55.6 \%$ in the 2-point group, respectively.

Conclusion: Fib-4 index and previous HCC history were independent predictors for development of HCC after DAA treatment. Patients with these risk factors require careful observation.

Keywords: hepatocellular carcinoma, antiviral agents, sustained virological response

\section{Introduction}

Hepatitis $\mathrm{C}$ is a global health issue, and patients with hepatitis $\mathrm{C}$ virus (HCV) infection are at risk of progressive liver disease, which ultimately leads to cirrhosis and hepatocellular carcinoma (HCC). ${ }^{1}$ The therapeutic goal is to eliminate $\mathrm{HCV}$ and then prevent progression to hepatic cirrhosis and/or development of HCC, thus improving the patient's prognosis. Obtaining sustained virologic response (SVR) with interferon (IFN)-based therapy could reduce the HCC occurrence rate post$\mathrm{SVR}^{2,3}$

Currently, direct-acting antiviral agents (DAA), which inhibit HCV replication, are standard treatment for chronic $\mathrm{HCV}$ infection, with combinations of nonstructural protein (NS) 3/4A protease, NS5A and NS5B polymerase inhibitors being used in treatment. Treatment duration has also become shorter. Oral IFNfree treatment enables not only chronic hepatitis patients but also those with decompensated cirrhosis to achieve high SVR rates with better safety than IFNbased therapy.
Correspondence: Akio Miyasaka Division of Hepatology, Department of Internal Medicine, Iwate Medical University School of Medicine, 2-I-I Idaidori, Yahaba-cho, Shiwa-gun, Iwate, 028-3695, Japan

Tel $+8|-| 9-6|3-7|||$

Fax +8I-19-907-7|66

Email akimiya@iwate-med.ac.jp 
Studies have also reported that eradication of HCV by DAA treatment reduced the risk of $\mathrm{HCC} .{ }^{4-6}$ However, it is unclear if $\mathrm{HCC}$ occurrence will decline and whether there are risk factors related to HCC over the long term after elimination of $\mathrm{HCV}$.

This study aimed to investigate the long-term incidence of HCC after successful DAA treatment and to evaluate the factors associated with HCC occurrence using pretreatment data.

\section{Materials and Methods}

\section{Study Design and Patient Population}

This study was a retrospective cohort study. A total of 467 consecutive patients with chronic HCV infection who had chronic hepatitis or hepatic cirrhosis were treated with DAA between December 2014 and August 2021 at Iwate Medical University Hospital and an affiliated institution.

The presence of cirrhosis was investigated at screening based on various combinations of liver biopsy findings, a fibrosis-4 (Fib-4) index ${ }^{7}$ of $\geq 3.25$, serum markers of fibrosis, transient elastography and a liver imaging examination (ultrasonography [US], computed tomography $[\mathrm{CT}]$ or magnetic resonance imaging [MRI]), as well as the patient's clinical state.

Patients with HCV genotype 1 were treated with 24 weeks of asunaprevir plus daclatasvir, or 12 weeks of ledipasvir/sofosbuvir, ombitasvir/paritaprevir/ritonavir, grazoprevir/elbasvir, and asunaprevir/daclatasvir/beclabuvir. Patients with HCV genotype 2 were administered sofosbuvir and ribavirin and ledipasvir/sofosbuvir for 12 weeks. Patients with HCV genotype $1 / 2$ were treated with glecaprevir/pibrentasvir for $8-12$ weeks. Decompensated cirrhotic patients with $\mathrm{HCV}$ genotype $1 / 2$ received 12 weeks of velpatasvir/sofosbuvir treatment.

SVR was defined as undetectable HCV RNA at 12 weeks after treatment completion.

Diabetes mellitus (DM) was defined as DM pattern in $75 \mathrm{~g}$ oral glucose tolerance test, HbAlc $>6.5 \%$ or taking anti-diabetes drugs and/or injecting insulin before starting DAA therapy.

This study was performed in accordance with the Declaration of Helsinki and was also approved by the ethics committee of Iwate Medical University (approval number H26-75 and H27-135 and 147) and the relevant committees at the treatment site. Written informed consent was obtained from each patient before treatment.

\section{Clinical and Laboratory Assessment}

Clinical and laboratory assessments were carried out before treatment, at end of treatment (EOT), at 24 weeks after EOT, and thereafter at 3-6 months. Serum HCV RNA levels were measured using a COBA TaqMan HCV quantitation assay (Roche Diagnostics, Tokyo, Japan), with a detection range of $15-6.9 \times 10^{7} \mathrm{IU} / \mathrm{mL}(1.2-7.8 \mathrm{log}$ $\mathrm{IU} / \mathrm{mL}$ ), and undetectable HCV RNA was defined as negative. HCV genotype was determined by polymerase chain reaction as previously defined by Okamoto et al. ${ }^{8}$

Fib-4 index, considered to be a surrogate marker of liver fibrosis, was calculated based on the following formula: Fib-4 index $=($ aspartate aminotransferase $[\mathrm{AST}] \times$ age $) /($ platelet $\times$ Valanine aminotransferase $[$ ALT] $)$.

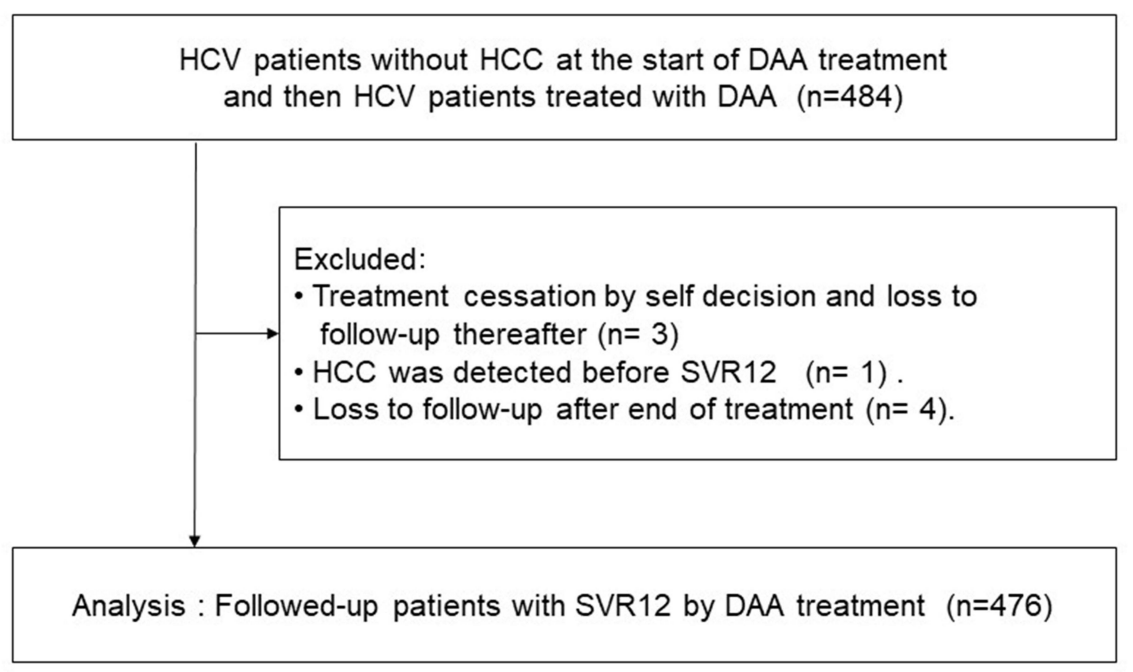

Figure I Flowchart depicting selection of participant. 


\section{Follow Up and Diagnosis with HCC}

HCC surveillance was conducted every 3-6 months by blood test including $\alpha$-fetoprotein (AFP) and imaging examination such as US, CT and MRI. Imaging examination was performed every 6 months for patients with chronic hepatitis and every 3 months for patients with liver cirrhosis (LC). HCC was diagnosed histologically and/or based on imaging findings.
We identified all new HCC cases diagnosed at least 12 weeks after the initiation of DAA treatment.

\section{Statistical Analysis}

Categorical variables were analyzed using the Chi-square test and Fisher's exact test, and continuous variables were analyzed using the Mann-Whitney $U$-test to compare clinical parameters among the groups. The HCC incidence rate was

Table I Baseline Characteristics of Patients Who Obtained SVR with DAA Treatment

\begin{tabular}{|c|c|c|c|c|}
\hline & Overall $(n=476)$ & $\operatorname{HCC}(+)(n=40)$ & HCC $(-)(n=436))$ & p-value HCC (+) vs HCC (-) \\
\hline Age (years) ${ }^{\#}$ & $68(21-90)$ & $70(55-82)$ & $67(21-90)$ & 0.0533 \\
\hline Sex (male/female) ${ }^{\#}$ & $227 / 249$ & $30 / 10$ & $197 / 239$ & 0.0004 \\
\hline Body mass index $\left(\mathrm{kg} / \mathrm{m}^{2}\right)^{\#}$ & $23.1(14.4-36.6)$ & $24.8(15.8-33.1)$ & $22.9(14.4-36.6)$ & 0.1540 \\
\hline Liver status (CH/LC) & $334 / 142$ & $12 / 28$ & $322 / 114$ & $<0.0001$ \\
\hline DAA treatment & $\begin{array}{l}\text { ASV/DCV II0, } \\
\text { LDV/SOF II9, } \\
\text { SOF/RBV 65, } \\
\text { PTV/OBV/r II, } \\
\text { ASV/DCV /BEC I, } \\
\text { EBR/GZR 69, } \\
\text { GLE/PIB 73, } \\
\text { SOF/VEL 24, } \\
\text { SOF/VEL/RBV } 4\end{array}$ & $\begin{array}{l}\text { ASV/DCV I3, } \\
\text { LDV/SOF 7, } \\
\text { SOF/RBV 4, } \\
\text { PTV/OBV/r 0, } \\
\text { ASV/DCV /BEC 0, } \\
\text { EBR/GZR 9, } \\
\text { GLE/PIB I, } \\
\text { SOF/VEL 5, } \\
\text { SOF/VEL/RBV I }\end{array}$ & $\begin{array}{l}\text { ASV/DCV 97, } \\
\text { LDV/SOF II2, } \\
\text { SOF/RBV 6I, } \\
\text { PTV/OBV/r II, } \\
\text { ASV/DCV /BEC I, } \\
\text { EBR/GZR 60, } \\
\text { GLE/PIB 72, } \\
\text { SOF/VEL 19, } \\
\text { SOF/VEL/RBV } 3\end{array}$ & \\
\hline Total bilirubin (mg/dL) ${ }^{\#}$ & $0.6(0.2-5.4)$ & $0.7(0.3-1.4)$ & $0.6(0.2-5.4)$ & 0.2750 \\
\hline Albumin $(\mathrm{g} / \mathrm{dL}){ }^{\#}$ & $4.1(2.4-5.2)$ & $3.8(3.1-4.7)$ & $4.1(2.4-5.2)$ & 0.0002 \\
\hline Prothrombin activity (\%) ${ }^{\#}$ & $97(21-100)$ & $89(21-100)$ & $98(24-100)$ & 0.0009 \\
\hline AST $(\mathrm{U} / \mathrm{L}){ }^{\#}$ & $36(12-164)$ & $51(19-148)$ & $35(12-164)$ & $<0.0001$ \\
\hline $\operatorname{ALT}(\mathrm{U} / \mathrm{L}){ }^{\#}$ & $34(8-237)$ & $52(16-180)$ & $34(8-237)$ & 0.0024 \\
\hline White blood cells $(/ \mu \mathrm{L}){ }^{\#}$ & $4580(1010-13,110)$ & $3995(1650-6760)$ & $4640(1010-13,110)$ & 0.0039 \\
\hline Hemoglobin $(g / d L) ~ \#$ & $13.2(8.6-17.7)$ & $12.7(10.3-16.4)$ & $13.2(8.6-17.7)$ & 0.1630 \\
\hline Platelet count $\left(\times 10^{4} / \mu \mathrm{L}\right){ }^{\#}$ & $15.0(2.6-44.2)$ & II.8 (2.6-22.5) & $15.3(3.0-44.2)$ & $<0.0001$ \\
\hline LDL-cholesterol $(\mathrm{mg} / \mathrm{dL}){ }^{\#}$ & $88(20-173)$ & $81(37-123)$ & $90(20-173)$ & 0.0402 \\
\hline Fib-4 index $\#$ & $2.88(0.32-24.5 \mathrm{I})$ & $4.7 \mid(2.23-18.78)$ & $2.75(0.32-24.5 \mathrm{I})$ & $<0.0001$ \\
\hline $\operatorname{AFP}(\mathrm{ng} / \mathrm{mL}){ }^{\#}$ & $4.1(0.7-141.7)$ & $9.6(1.7-141.7)$ & $3.9(0.7-114.4)$ & $<0.0001$ \\
\hline HCV RNA (Log IU/mL) ${ }^{\#}$ & $6.1(2.7-7.3)$ & $5.8(3.9-6.9)$ & $6.1(2.9-7.3)$ & 0.1200 \\
\hline Diabetes mellitus (yes/no) & $128 / 348$ & $16 / 24$ & $112 / 324$ & 0.0854 \\
\hline Previous HCC treatment (yes/no) & $61 / 415$ & $28 / 12$ & $33 / 403$ & $<0.0001$ \\
\hline Observation period (months) & $46.4(3.5-75.7)$ & $17.8(4.9-62.1)$ & $50.0(3.5-75.7)$ & $<0.0001$ \\
\hline
\end{tabular}

Note: "Data are expressed as median (range).

Abbreviations: HCV, hepatitis C virus; DAAs, direct-acting antiviral agent; SVR, sustained virological response; ASV, asunaprevir; DCV, daclatasvir; LDV, ledipasvir; SOF, sofosbuvir; RBV, ribavirin; PTV, paritaprevir; OMV, ombitasvir; r, ritonavir; BEC, beclabuvir; EBR, elbasvir; GRZ, grazoprevir; GLE, glecaprevir; PIB, pibrentasvir; VEL, velpatasvir; $\mathrm{CH}$, chronic hepatitis; LC, liver cirrhosis; AST, aspartate aminotransferase; ALT, alanine aminotransferase; LDL, low-density lipoprotein; Fib-4, index, fibrosis-4 index; AFP, alpha-fetoprotein; HCC, hepatocellular carcinoma. 
calculated using the Kaplan-Meier method, and the Log rank test was conducted to evaluate the differences between the curves. Multivariate Cox hazard regression analysis was performed to predict significant factors related to $\mathrm{HCC}$ development. Factors that were statistically significant in the univariate analysis were entered into the multivariate Cox hazard regression analysis to identify significant independent factors after conversion to categorical data of two ordinal numbers. The median value of each factor was set as the cutoff point. Potential confounding factors associated with liver fibrosis were excluded. Hazard ratios (HRs) and 95\% confidence intervals (CIs) were also calculated. $P$-values of $<0.05$ (determined by two-tailed test) were considered statistically significant. The sensitivity, specificity, and likelihood ratio for positive results of the Fib-4 index were assessed in a receiver operating characteristic (ROC) curve. All statistical analyses were conducted using SPSS software (SPSS Statistics for Windows, SPSS Inc., Chicago, IL).

\section{Results}

\section{Patients' Characteristics}

Patient selection is depicted in Figure 1. In total, $484 \mathrm{HCV}$ patients, who underwent DAA treatment and obtained SVR, were enrolled. A total of eight patients were excluded because of treatment cessation by self-decision and loss to follow up thereafter $(\mathrm{n}=3)$, loss to follow up after EOT $(\mathrm{n}=$ $4)$, and HCC occurred before SVR12 $(n=1)$. Finally, a total of 476 patients with chronic HCV infection were analyzed.

Patient characteristics at baseline are shown in Table 1. The median age was 68 years (21-90), with 227 men and 249 women included. The study cohort comprised 334 participants with chronic hepatitis and 142 LC patients. The median observation period was 46.4 months (3.575.7) from 12 weeks post-EOT.

\section{Overall HCC Incidence Rate}

HCC developed in 40 patients during the study period. Therefore, the overall $\mathrm{HCC}$ incidence rate was $8.4 \%$. The incidence of $\mathrm{HCC}$ per 100 person-years was 2.35. The incidence rates of $\mathrm{HCC}$ were $3.7 \%, 6.0 \%, 7.1 \%, 9.3 \%$, and $10.6 \%$ at $1,2,3,4$, and 5 years post-SVR, respectively (Figure 2). The number of patients followed up at each time point is presented in Figure 2. Comparison of baseline characteristics between the HCC incidence and non-HCC incidence groups is shown in Table 1. Significant differences were found in sex, liver status, albumin, prothrombin activity, AST, ALT, white blood cell (WBC) count, platelet count,

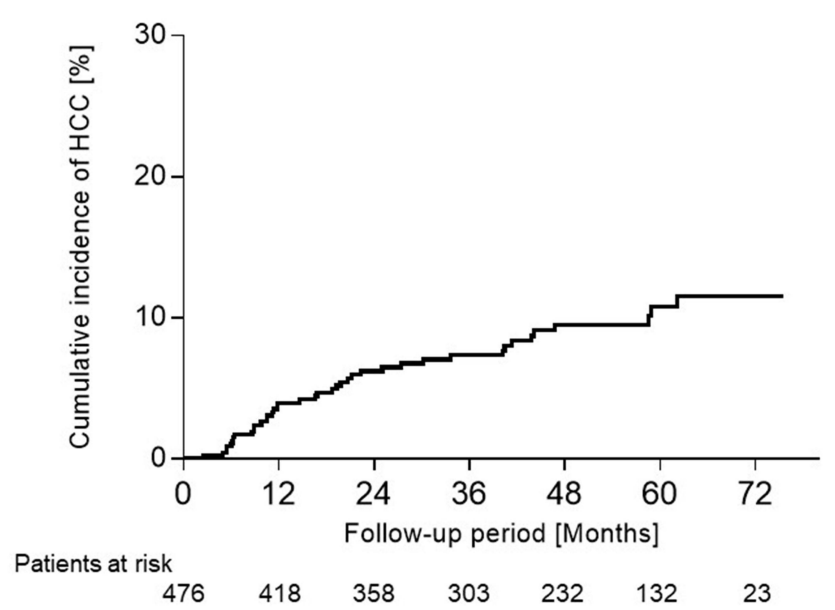

Figure 2 Cumulative incidence of hepatocellular carcinoma in patients who achieved hepatitis $\mathrm{C}$ virus (HCV) eradication by direct acting antivirals (DAAs).

low-density lipoprotein (LDL)-cholesterol, Fib-4 index, AFP, and previous HCC history between the groups.

\section{Prediction for HCC Development After HCV Elimination}

Pre-treatment factors that may contribute to HCC development after HCV elimination were evaluated by multivariate Cox hazard regression analysis. Sex, liver status, albumin, prothrombin activity, AST, WBC count, platelet count, lowdensity lipoprotein cholesterol, Fib-4 index, AFP, DM, and previous $\mathrm{HCC}$ history were significant risk factors related to development of HCC by univariate analysis. These significant factors were entered into the multivariate analysis, which identified that platelet counts $(\mathrm{HR}=0.24 ; 95 \% \mathrm{CI}$, 0.07-0.89, $P=0.0323)$, AFP (HR=3.14; 95\% CI, 1.02-9.67, $P=0.0468)$, Fib-4 index $(\mathrm{HR}=8.56 ; 95 \% \mathrm{CI}, 1.81-40.80$, $P=0.0069)$, and previous HCC history $(\mathrm{HR}=29.90 ; 95 \% \mathrm{CI}$, 7.21-125.50, $P<0.0001)$ were independent risk factors for incidence of HCC post-SVR (Table 2).

Regarding Fib-4 index, we performed ROC curve analysis to determine an appropriate cut-off value for incidence of HCC. Accordingly, 3.07 was applied as the best cut-off value before DAA treatment (Figure 3).

\section{Subgroup Analysis of HCC Occurrence}

The incidence of $\mathrm{HCC}$ was investigated regarding pretreatment factors contributing to $\mathrm{HCC}$ occurrence after DAA treatment that were extracted in the multivariate analysis, namely, sex, body mass index (BMI), prothrombin activity, platelet counts, AFP, Fib-4 index, and 
Table 2 Predictive Factors of HCC Development After Successful DAA Treatment in Patients with HCV Infection

\begin{tabular}{|c|c|c|c|c|c|c|c|}
\hline \multirow[t]{2}{*}{ Factor } & \multirow[t]{2}{*}{ Category } & \multicolumn{3}{|c|}{ Univariate Analysis } & \multicolumn{3}{|c|}{ Multivariate Analysis } \\
\hline & & HR & $95 \% \mathrm{Cl}$ & p-value & HR & $95 \% \mathrm{Cl}$ & p-value \\
\hline Age (years) & $\begin{array}{l}<68 \\
\geq 68\end{array}$ & $\begin{array}{l}1 \\
1.92\end{array}$ & $1.00-3.73$ & 0.0526 & & & \\
\hline Sex & $\begin{array}{l}\text { Female } \\
\text { Male }\end{array}$ & $\begin{array}{l}1 \\
3.55\end{array}$ & I.74-7.27 & 0.0005 & & & \\
\hline Liver status & $\begin{array}{l}\mathrm{CH} \\
\mathrm{LC}\end{array}$ & $\begin{array}{l}1 \\
6.37\end{array}$ & $3.23-12.56$ & $<0.0001$ & & & \\
\hline Albumin $(\mathrm{g} / \mathrm{dL})$ & $\begin{array}{l}\geq 4.1 \\
<4.1\end{array}$ & $\begin{array}{l}1 \\
3.00\end{array}$ & $1.52-5.90$ & 0.0015 & & & \\
\hline Prothrombin activity (\%) & $\begin{array}{l}\geq 97 \\
<97\end{array}$ & $\begin{array}{l}1 \\
2.54\end{array}$ & $1.30-4.95$ & 0.0062 & & & \\
\hline AST (U/L) & $\begin{array}{l}<36 \\
\geq 36\end{array}$ & $\begin{array}{l}1 \\
4.08\end{array}$ & $1.88-8.85$ & 0.0004 & & & \\
\hline ALT (U/L) & $\begin{array}{l}<34 \\
\geq 34\end{array}$ & $\begin{array}{l}1 \\
0.96\end{array}$ & $0.49-1.90$ & 0.9049 & & & \\
\hline White blood cells $(/ \mu \mathrm{L})$ & $\begin{array}{l}<4580 \\
\geq 4580\end{array}$ & $\begin{array}{l}1 \\
2.35\end{array}$ & $1.20-4.62$ & 0.0273 & & & \\
\hline Platelet count $\left(\times 10^{4} / \mu \mathrm{L}\right)$ & $\begin{array}{l}\geq 15.0 \\
<15.0\end{array}$ & $\begin{array}{l}1 \\
3.12\end{array}$ & $1.52-6.38$ & 0.0018 & $\begin{array}{l}1 \\
0.24\end{array}$ & $0.07-0.89$ & 0.0323 \\
\hline LDL- cholesterol (mg/dL) & $\begin{array}{l}<88 \\
\geq 88\end{array}$ & $\begin{array}{l}\text { I } \\
0.37\end{array}$ & $0.17-0.85$ & 0.0186 & & & \\
\hline Fib-4 index & $\begin{array}{l}<2.88 \\
\geq 2.88\end{array}$ & $\begin{array}{l}1 \\
9.18\end{array}$ & $3.26-25.78$ & $<0.0001$ & $\begin{array}{l}1 \\
8.56\end{array}$ & $1.81-40.80$ & 0.0069 \\
\hline $\operatorname{AFP}(\mathrm{ng} / \mathrm{mL})$ & $\begin{array}{l}<4.1 \\
\geq 4.1\end{array}$ & $\begin{array}{l}1 \\
5.35\end{array}$ & $2.37-12.89$ & $<0.000$ I & $\begin{array}{l}1 \\
3.14\end{array}$ & $1.02-9.67$ & 0.0468 \\
\hline Diabetes mellitus & $\begin{array}{l}\text { No } \\
\text { Yes }\end{array}$ & $\begin{array}{l}1 \\
1.90\end{array}$ & $1.01-3.57$ & 0.0476 & & & \\
\hline Previous $\mathrm{HCC}$ treatment & $\begin{array}{l}\text { No } \\
\text { Yes }\end{array}$ & $\begin{array}{l}1 \\
19.86\end{array}$ & $10.05-39.27$ & $<0.0001$ & $\begin{array}{l}1 \\
29.90\end{array}$ & $7.21-125.50$ & $<0.0001$ \\
\hline
\end{tabular}

Abbreviations: $\mathrm{HCC}$, hepatocellular carcinoma; $\mathrm{HCV}$, hepatitis $\mathrm{C}$ virus; $\mathrm{DAAs}$, direct-acting antiviral agent; $\mathrm{HR}$, hazard ratio; $95 \% \mathrm{Cl}$, $95 \%$ confidence interval; $\mathrm{CH}$, chronic hepatitis; LC, liver cirrhosis; AST, aspartate aminotransferase; ALT, alanine aminotransferase; LDL, low-density lipoprotein; Fib-4, index, fibrosis-4 index; AFP, alphafetoprotein.

previous HCC history. Of these factors, previous HCC history was the most significant factor, followed by Fib-4 index.

In the Log rank test, the cumulative HCC incidence was significantly higher in patients with previous $\mathrm{HCC}$ history than in patients without previous HCC history $(P<0.0001)$. The 1-, 2-, 3-, 4-, and 5-year incidence rates of HCC for patients with previous HCC history were $23.0 \%, 31.9 \%, 37.6 \%, 46.7 \%$, and $55.6 \%$, respectively, compared with $0.8 \%, 1.9 \%, 2.2 \%, 3.1 \%$, and $3.1 \%$, respectively, among patients without previous HCC history (Figure 4A). Characteristics at baseline in patients with and without previous history of HCC are shown in Table 3. Patients with previous HCC history were significantly older, more predominantly male and demonstrated more liver disease progression compared with those without previous HCC history.

Similarly, the cumulative HCC incidence was significantly higher in patients whose Fib-4 index was $\geq 3.07$ than in patients whose Fib-4 index $<3.07(P<0.05)$. The 1-, 2-, 


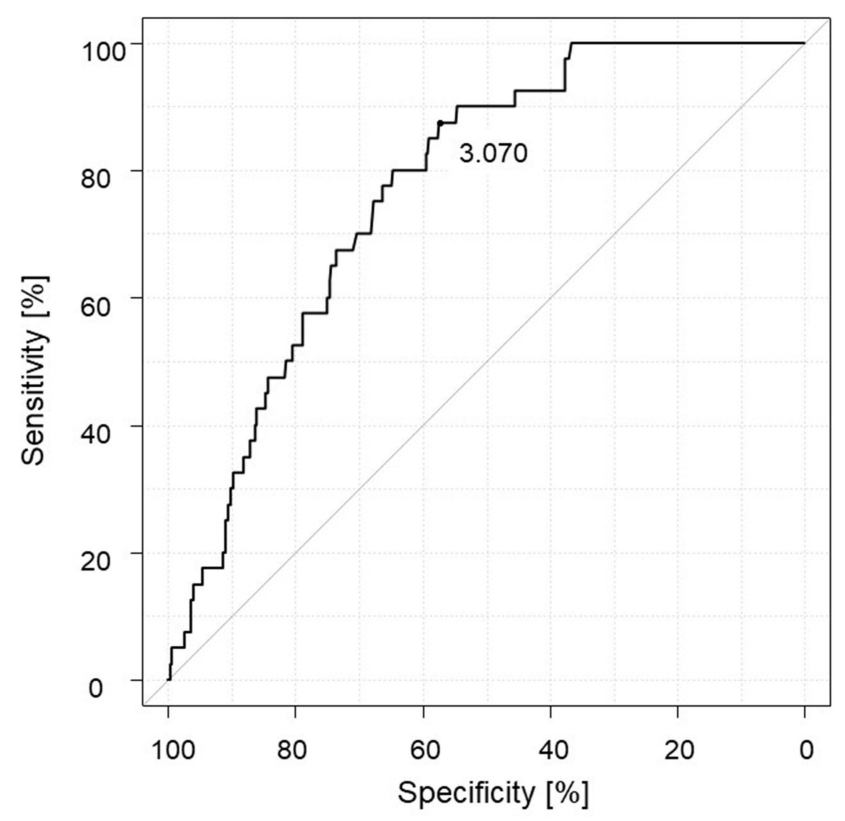

Figure 3 Receiver operating characteristic (ROC) curve of Fib-4 index for hepatocellular carcinoma (HCC) development.

3-, 4-, and 5-year incidence rates of HCC for patients with Fib-4 index $\geq 3.07$ were $6.6 \%, 11.0 \%, 13.3 \%, 16.9 \%$, and $19.6 \%$, respectively, compared with $1.3 \%, 1.7 \%, 1.7 \%$, $2.4 \%$, and 2.4, respectively, among patients with Fib-4 index $<3.07$ (Figure 4B).

\section{Prediction of HCC Occurrence Based on Stratified Factors}

On the basis of the results of the multivariate analysis that included pre-treatment factors, a scoring system combining the Fib-4 index and previous HCC history was developed. First, previous HCC history was divided into presence and absence. Presence of previous HCC history was scored as 2. Next, absence of previous HCC history was stratified into two groups by Fib-4 index. Thus, Fib-4 index values of $<3.07$ and $\geq 3.07$ were scored as 0 and 1 , respectively. The scores combining previous HCC history and Fib-4 index were calculated. Of the study patients with the scores calculated above, the study patients were then grouped based on those scores as follows: 0-point group $(\mathrm{n}=245), 1$-point group $(\mathrm{n}=169)$, and 2-point group $(\mathrm{n}=$ 62). Figure 5 indicates the cumulative HCC incidence stratified by score. The 1-, 2-, 3-, 4-, and 5-year incidence rates of $\mathrm{HCC}$ were $0.4 \%, 0.4 \%, 0.4 \%, 0.4 \%$, and $0.4 \%$, respectively, in the 0 -point group; $1.2 \%, 4.1 \%, 4.9 \%$, $6.8 \%$, and $6.8 \%$, respectively, in the 1-point group; and $23.0 \%, 31.9 \%, 37.6 \%, 46.7 \%$, and $55.6 \%$, respectively, in

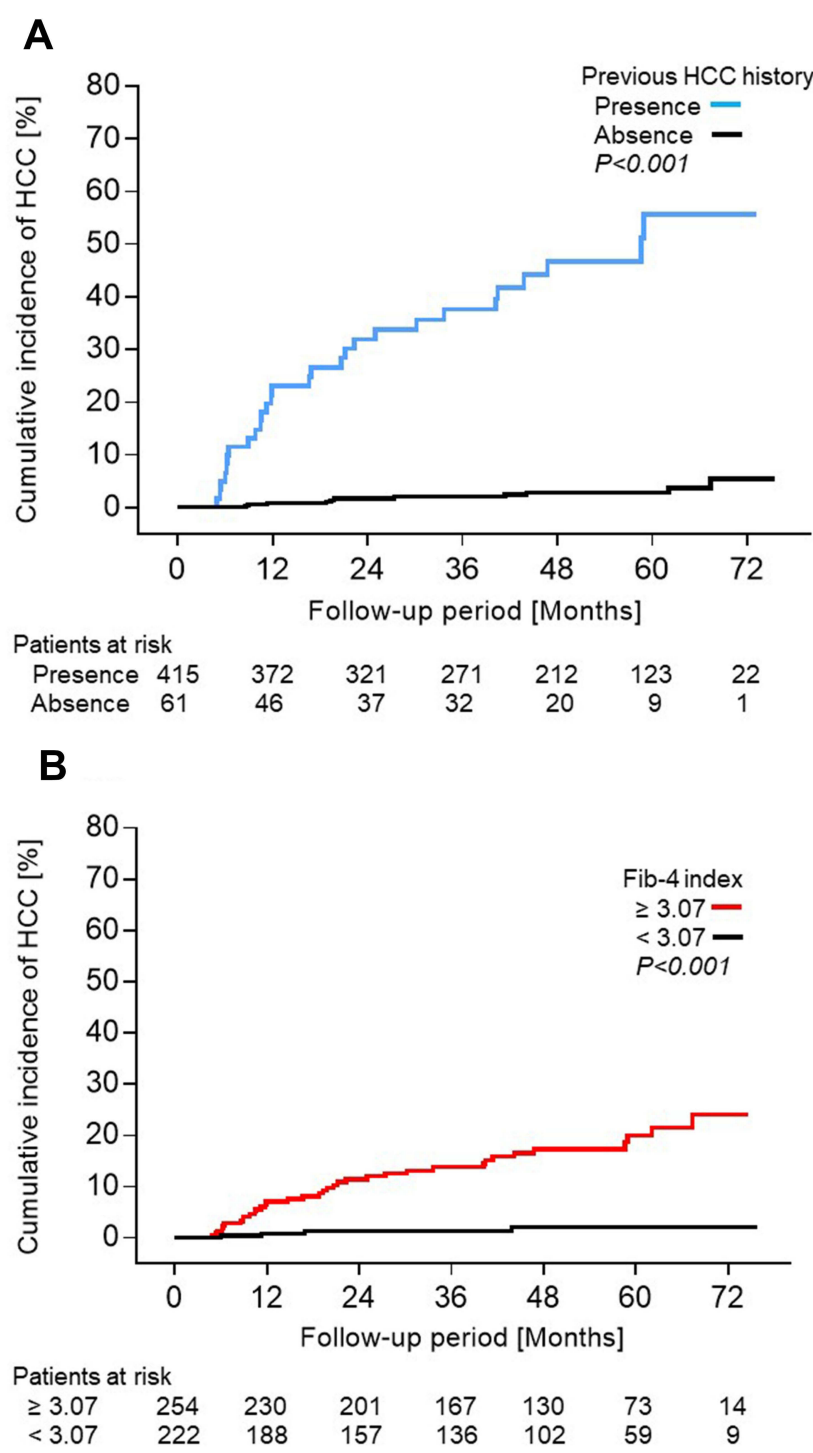

Figure 4 The cumulative hepatocellular carcinoma (HCC) incidence stratified according to the factors that might contribute to the development of HCC (A) Presence of previous HCC history (blue line), absence of previous HCC history (black line). (B) Fib-4 index $\geq 3.07$ (red line), Fib-4 index $<3.07$ (black line).

the 2-point group. The cumulative HCC incidence increased significantly in the 2 -point group $(P<0.05)$.

\section{Discussion}

Oral IFN-free treatment has allowed achievement of high SVR rates with improved safety compared with IFN-based treatments. Moreover, it has become possible to safely treat elderly and cirrhotic patients, which was previously difficult using IFN treatment regimens.

Many reports have investigated hepatic carcinogenesis after SVR with IFN-based treatment. In these reports, elderly age, male sex, presence of fatty liver, advanced liver fibrosis, 
Table 3 Baseline Characteristics of Patients with and without Previous History of HCC

\begin{tabular}{|c|c|c|c|}
\hline & Previous HCC History $(n=6 I)$ & Non Previous HCC History $(n=4 \mid 5)$ & P-value \\
\hline Age (years) ${ }^{\#}$ & $70(56-90)$ & $67(21-86)$ & 0.0014 \\
\hline Sex (male/female) & $42 / 19$ & $185 / 230$ & 0.0005 \\
\hline Body mass index $\left(\mathrm{kg} / \mathrm{m}^{2}\right)^{\#}$ & $23.5(15.8-34.6)$ & $22.9(14.4-36.6)$ & 0.2760 \\
\hline Liver status (CH/LC) & $11 / 50$ & $323 / 92$ & $<0.0001$ \\
\hline Total bilirubin $(\mathrm{mg} / \mathrm{dL}){ }^{\#}$ & $0.7(0.4-2.9)$ & $0.6(0.2-5.4)$ & 0.0614 \\
\hline Albumin $(\mathrm{g} / \mathrm{dL}){ }^{\#}$ & $3.7(3.0-5.0)$ & $4.1(2.4-5.2)$ & $<0.0001$ \\
\hline Prothrombin activity (\%) \# & $86(21-100)$ & $98(24-100)$ & $<0.0001$ \\
\hline AST $(\mathrm{U} / \mathrm{L}){ }^{\#}$ & $49(18-148)$ & $35(12-164)$ & 0.0001 \\
\hline ALT $(\mathrm{U} / \mathrm{L}){ }^{\#}$ & $39(16-180)$ & $34(8-237)$ & 0.0064 \\
\hline White blood cells $(/ \mu \mathrm{L}){ }^{\#}$ & $4090(1350-6360)$ & $4650(1010-13,110)$ & 0.0028 \\
\hline Hemoglobin $(\mathrm{g} / \mathrm{dL}){ }^{\#}$ & $12.8(9.9-16.0)$ & $13.3(8.6-17.7)$ & 0.0236 \\
\hline Platelet count $\left(\times 10^{4} / \mu \mathrm{L}\right){ }^{\#}$ & II.2(2.6-22.5) & $15.5(3.0-44.2)$ & $<0.0001$ \\
\hline LDL-cholesterol (mg/dL) $\#$ & $81(30-123)$ & $90(20-173)$ & 0.0116 \\
\hline Fib-4 index $\#$ & $4.48(1.68-19.66)$ & $2.68(0.32-24.5 \mathrm{I})$ & $<0.0001$ \\
\hline $\operatorname{AFP}(\mathrm{ng} / \mathrm{mL}) \#$ & $8.9(1.7-|4| .7)$ & $3.9(0.7-112.7)$ & $<0.0001$ \\
\hline HCV RNA (Log IU/mL) ${ }^{\#}$ & $5.8(2.9-7.3)$ & $6.1(2.7-7.3)$ & 0.0186 \\
\hline Diabetes mellitus (yes/no) & $28 / 33$ & $100 / 315$ & 0.0006 \\
\hline Observation period (months) & $38.2(4.9-73.2)$ & $49.0(3.5-75.7)$ & 0.0042 \\
\hline HCC occurrence (yes/no) & $28 / 33$ & $12 / 403$ & $<0.0001$ \\
\hline
\end{tabular}

Note: ${ }^{\#}$ Data are expressed as median (range).

Abbreviations: HCC, hepatocellular carcinoma; $\mathrm{CH}$, chronic hepatitis; LC, liver cirrhosis; AST, aspartate aminotransferase; ALT, alanine aminotransferase; LDL, low-density lipoprotein; Fib-4, index, fibrosis-4 index; AFP, alpha-fetoprotein; $\mathrm{HCV}$, hepatitis $\mathrm{C}$ virus.

DM, alcohol consumption, and AFP and ALT elevation were listed as contributing factors to HCC occurrence., $2,3,9-12$ Moreover, several studies reported HCC occurrence after $\mathrm{SVR}$ in chronic hepatitis $\mathrm{C}$ patients treated with IFN-based

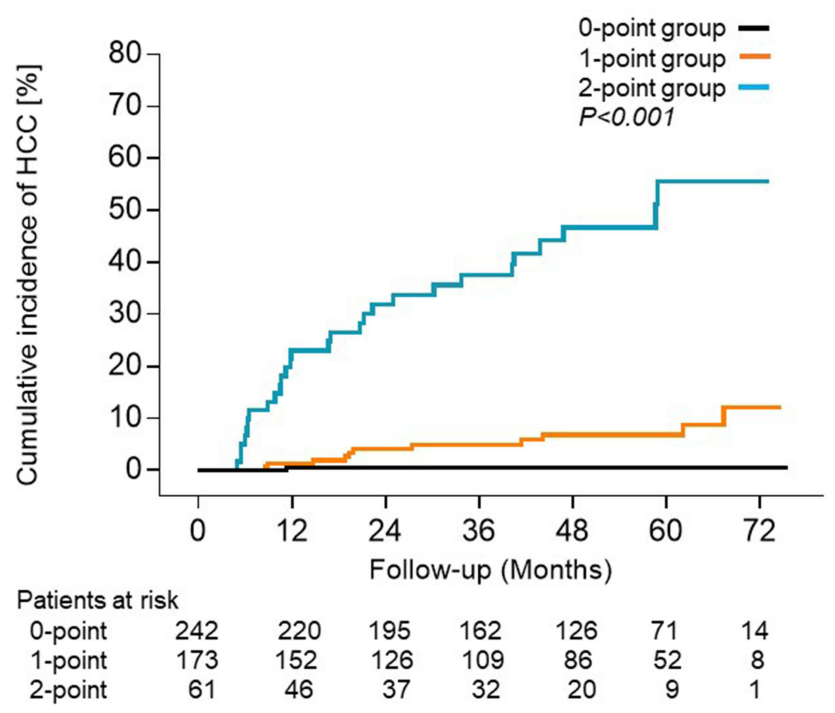

Figure 5 Cumulative incidence of hepatocellular carcinoma $(\mathrm{HCC})$ by the score combining the Fib-4 index and previous HCC history Presence of previous HCC history; 2-point group (blue line), absence of previous HCC history and Fib-4 index $\geq 3.07$; I-point group (orange line), absence of previous HCC history and Fib-4 index <3.07; 0-point group (black line). treatment compared with those treated with DAAs. ${ }^{13-15}$ These studies demonstrated that the incidence of HCC development after HCV eradication was similar between patients receiving IFN-based or DAA treatment.

In this study, we observed long-term cumulative $\mathrm{HCC}$ incidence after successful DAA treatment. As a result, the incidence rates of $\mathrm{HCC}$ were $3.7 \%, 6.0 \%, 7.1 \%, 9.3 \%$, and $10.6 \%$ at $1,2,3,4$, and 5 years post-SVR 12 , respectively. The cumulative HCC incidence in this study tended to be higher than that in other studies. ${ }^{4-6,16}$ Because the incidence of $\mathrm{HCC}$ in patients without previous HCC history was low, previous HCC history may be a contributing factor to high HCC development. Moreover, this study included decompensated LC. Therefore, this study more closely reflects the real clinical setting.

One aim of this study was to explore the factors associated with HCC occurrence. Other studies reported that age, sex, platelet count, gamma-glutamyl transpeptidase, Fib-4 index, albumin-bilirubin grade, and DM were risk factors related to incidence of HCC after SVR by DAA treatment. ${ }^{4-6,16}$ In our study, sex, liver status, albumin, prothrombin activity, AST, WBC count, platelet count, Fib-4 index, AFP, DM, and previous $\mathrm{HCC}$ history were extracted as risk factors related to development of HCC by univariate analysis, and platelet count, AFP, Fib-4 index, and previous HCC history were 
extracted by multivariate analysis. Of these factors, previous HCC history was the most significant, followed by Fib-4 index. HCC recurrence was observed at high probability in patients with previous HCC history after SVR by DAA treatment. The recurrence of HCC after DAA treatment and SVR in patients with previous HCC history was reported. ${ }^{17-20}$ In these reports, patients with previous HCC history included those with advanced liver fibrosis, older and male individuals compared with patients without previous HCC history. Similar results were seen in our study. Fib-4 index is calculated by patient's age, AST, ALT, and platelet levels, and is an easy-touse non-invasive marker of liver fibrosis. Fib-4 index has been shown to be associated with HCC risk in non-cirrhotic and cirrhotic HCV patients treated with DAA. ${ }^{21,22}$ The Fib-4 index, considered to reflect liver fibrosis in patients with $\mathrm{HCV}$, was also extracted in this study, and HCC occurrence was high in patients with high Fib-4 index. However, the cutoff value of the Fib-4 index is different among reports. In this study, 3.07 was applied as the cut-off value for Fib-4 index, and ROC analysis of our study population indicated that sensitivity was $87.2 \%$ and specificity was $57.3 \%$ at this cutoff value. Furthermore, several reports have investigated prediction of HCC occurrence after successful DAA treatment using combinations of stratified factors that contribute to HCC occurrence. ${ }^{4-6,16}$ Here, however, we stratified by Fib-4 index and previous HCC history, and then predicted HCC incidence using these stratification factors. Resultingly, incidence of HCC in patients with previous HCC history was significantly high. Regarding patients without previous HCC history, incidence of HCC in patients with Fib-4 index $\geq 3.07$ was significantly higher compared with that in patients with $<3.07$. Therefore, it is necessary to carefully follow up after SVR by DAA treatment in patients with previous HCC history, and in Fib-4 index $\geq 3.07$ patients without previous HCC history.

This study had several limitations. First, the sample population was relatively small. Second, only pretreatment parameters were used. However, clinical tests are routinely performed at initiation of treatment, and treatment influences the outcome of many of these tests. Third, the impact of fatty liver and alcohol consumption could not be sufficiently evaluated.

\section{Conclusions}

In conclusion, Fib-4 index and previous HCC history were independent predictors for developing HCC after DAA treatment. Patients with these risk factors require careful ongoing observation.

\section{Acknowledgments}

The authors express their sincere gratitude to Junko Sasaki for her help and support. We thank the collaborators at the participating centers. We also thank Gillian Campbell, $\mathrm{PhD}$ from Edanz for editing drafts of this manuscript.

\section{Author Contributions}

Conceptualization, Akio Miyasaka; data curation, all authors; formal analysis, Akio Miyasaka; methodology, Akio Miyasaka; resource: all authors; supervision, Yasuhiro Takikawa; writing - original draft preparation, Akio Miyasaka; writing - review \& editing, all authors. All authors have read and approved the final version of the manuscript. Akio Miyasaka had full access to all of the data in this study and takes complete responsibility for the integrity of the data and the accuracy of the data analysis.

\section{Funding}

This study was partly supported by a grant from the Japanese Ministry of Health, Labor, and Welfare (19HC1001).

\section{Disclosure}

The authors have no conflicts of interest directly relevant to the content of this article.

\section{References}

1. Kiyosawa K, Sodeyama T, Tanaka E, et al. Interrelationship of blood transfusion, non-A, non-B hepatitis and hepatocellular carcinoma: analysis by detection of antibody to hepatitis $\mathrm{C}$ virus. Hepatology. 1990;12:671-675. doi:10.1002/hep.1840120409

2. Ikeda M, Fujiyama S, Tanaka M, et al. Risk factors for development of hepatocellular carcinoma in patients with chronic hepatitis $\mathrm{C}$ after sustained response to interferon. $J$ Gastroenterol. 2005;40:148-156. doi:10.1007/s00535-004-1519-2

3. Tokita H, Fukui H, Tanaka A, et al. Risk factors for the development of hepatocellular carcinoma among patients with chronic hepatitis $\mathrm{C}$ who achieved a sustained virological response to interferon therapy. J Gastroenterol Hepatol. 2005;20:752-758. doi:10.1111/ j.1440-1746.2005.03800.x

4. Watanabe T, Tokumoto Y, Joko K, et al. Predictors of hepatocellular carcinoma occurrence after direct-acting antiviral therapy in patients with hepatitis C virus infection. Hepatol Res. 2019;49:136-146. doi:10.1111/hepr.13278

5. Tani J, Morishita A, Sakamoto T, et al. Simple scoring system for prediction of hepatocellular carcinoma occurrence after hepatitis $\mathrm{C}$ virus eradication by direct-acting antiviral treatment: all Kagawa Liver Disease Group Study. Oncol Lett. 2020;19:2205-2212. doi:10.3892/ol.2020.11341

6. Ide T, Koga H, Nakano M, et al. Direct-acting antiviral agents do not increase the incidence of hepatocellular carcinoma development: a prospective, multicenter study. Hepatol Int. 2019;13:293-301. doi:10.1007/s12072-019-09939-2 
7. Vallet-Pichard A, Mallet V, Nalpas B, et al. FIB-4: an inexpensive and accurate marker of fibrosis in $\mathrm{HCV}$ infection. comparison with liver biopsy and fibrotest. Hepatology. 2007;46:32-36. doi:10.1002/ hep. 21669

8. Okamoto H, Kurai K, Okada S, et al. Full-length sequence of a hepatitis $\mathrm{C}$ virus genome having poor homology to reported isolates: comparative study of four distinct genotypes. Virology. 1992;188:331-341. doi:10.1016/0042-6822(92)90762-E

9. Tanaka A, Uegaki S, Kurihara H, et al. Hepatic steatosis as a possible risk factor for the development of hepatocellular carcinoma after eradication of hepatitis $\mathrm{C}$ virus with antiviral therapy in patients with chronic hepatitis C. World $J$ Gastroenterol. 2007;13:5180-5187. doi:10.3748/wjg.v13.i39.5180

10. Hirakawa M, Ikeda K, Arase Y, et al. Hepatocarcinogenesis following HCV RNA eradication by interferon in chronic hepatitis patients. Intern Med. 2008;47:1637-1643. doi:10.2169/ internalmedicine.47.1087

11. Arase Y, Kobayashi M, Suzuki F, et al. Effect of type 2 diabetes on risk for malignancies includes hepatocellular carcinoma in chronic hepatitis C. Hepatology. 2013;57:964-973. doi:10.1002/hep.26087

12. Asahina Y, Tsuchiya K, Nishimura T, et al. Alpha-fetoprotein levels after interferon therapy and risk of hepatocarcinogenesis in chronic hepatitis C. Hepatology. 2013;58:1253-1262. doi:10.1002/hep.26442

13. Nagaoki Y, Imamura M, Akita $H$, et al. The risk of hepatocellular carcinoma development after HCV eradication are similar between patients with peg-interferon plus ribavirin and direct-acting antiviral therapy. PLoS One. 2017;12:e0182710. doi:10.1371/journal. pone. 0182710

14. Kobayashi M, Suzuki F, Fujiyama S, et al. Sustained virologic response by direct antiviral agents reduces the incidence of hepatocellular carcinoma in patients with $\mathrm{HCV}$ infection. J Med Virol. 2017;89:476-483. doi:10.1002/jmv.24663

15. Tahata Y, Sakamori R, Urabe A, et al. Clinical outcomes of direct-acting antiviral treatments for patients with hepatitis $\mathrm{C}$ after hepatocellular carcinoma are equivalent to interferon treatment. Hepatol Res. 2020;50:1118-1233. doi:10.1111/hepr.13547
16. Abe K, Wakabayashi H, Nakayama H, et al. Factors associated with hepatocellular carcinoma occurrence after $\mathrm{HCV}$ eradication in patients without cirrhosis or with compensated cirrhosis. PLoS One. 2020;15:e0243473. doi:10.1371/journal.pone.0243473

17. Kanda T, Lau GKK, Wei L, et al. APASL HCV guidelines of virus-eradicated patients by DAA on how to monitor HCC occurrence and HBV reactivation. Hepatol Int. 2019;13:649-661. doi:10.1007/s12072-019-09988-7

18. Ikeda K, Kawamura Y, Kobayashi M, et al. Direct-acting antivirals decreased tumor recurrence after initial treatment of hepatitis $\mathrm{C}$ virus-related hepatocellular carcinoma. Dig Dis Sci. 2017;62:2932-2942. doi:10.1007/s10620-017-4739-Z

19. Nagaoki Y, Imamura $M$, Nishida $Y$, et al. The impact of interferon-free direct-acting antivirals on clinical outcome after curative treatment for hepatitis $\mathrm{C}$ virus-associated hepatocellular carcinoma: comparison with interferon-based therapy. J Med Virol. 2019;91:650-658. doi:10.1002/jmv.25352

20. Sugiura A, Joshita UT, Yamazaki T, et al. Past history of hepatocellular carcinoma is an independent risk factor of treatment failure in patients with chronic hepatitis $\mathrm{C}$ virus infection receiving direct-acting antivirals. J Viral Hepat. 2018;25:1462-1471. doi:10.1111/jvh.12973

21. Kawal F, Kramer JR, Asch SM, Chayanupatkul M, Cao Y, El-Serag HB. Risk of hepatocellular cancer in HCV patients treated with direct-acting antiviral agents. Gastroenterology. 2017;153:996-1005. doi:10.1053/j.gastro.2017.06.012

22. Ioannou GN, Beste LA, Green PK, Singal AG, Tapper EB, Waljee AK. Increased risk for hepatocellular carcinoma persists up to 10 years after $\mathrm{HCV}$ eradication in patients with baseline cirrhosis or high FIB-4 scores. Gastroenterology. 2019;157:1264-1278. doi:10.1053/j.gastro.2019.07.033
International Journal of General Medicine

\section{Publish your work in this journal}

The International Journal of General Medicine is an international, peer-reviewed open-access journal that focuses on general and internal medicine, pathogenesis, epidemiology, diagnosis, monitoring and treatment protocols. The journal is characterized by the rapid reporting of reviews, original research and clinical studies

\section{Dovepress}

across all disease areas. The manuscript management system is completely online and includes a very quick and fair peer-review system, which is all easy to use. Visit http://www.dovepress.com/ testimonials.php to read real quotes from published authors. 